\title{
Two Octaves Bandwidth Passive Balun for the Eleven Feed for Reflector Antennas
}

\author{
Abolghasem Zamanifekri ${ }^{(1)}$ and Jian Yang ${ }^{(1)}$ \\ (1) Depart. of Signals and Systems, Chalmers University of Technology, Sweden \\ E-mail: fekri@student.chalmers.se
}

\section{Introduction}

Wideband antenna technologies are in growing interest for future radio telescopes, such as in 1-10 GHz SKA (the squire kilometer array) [1] and 2-13 GHz VLBI 2010 (VLBI=Very Long Baseline Interferometry) [2] projects. The Eleven antenna, a new decade bandwidth log-periodic dual-dipole antenna developed at Chalmers University of technology, has very good features for applications in these radio telescopes due to the nearly constant beamwidth with about $11 \mathrm{dBi}$ directivity, the fixed phase center location over the whole bandwidth, low profile, cryogenic cool-ability, high $\mathrm{A} / \mathrm{T}$ values and simple geometry [3][4]. A dual polarized Eleven antenna is shown in Fig. 1.

One challenge in development of the decade bandwidth Eleven antenna is the decade bandwidth baluns which are required to feed it. There are three alternative solutions to decade bandwidth baluns [4]: using 180 deg hybrid as balun, active balun and passive balun. Decade bandwidth 180 deg hybrids are available commercially but they are bulky and lossy, which increases the system noise temperature in low noise radio telescopes. Differential low noise amplifiers (LNA) can be used as active baluns with low noise temperature [4]. However, because four differential LNAs are needed for the Eleven antenna, gain variation among the LNAs is a problem. Therefore, a compact, low loss and decade bandwidth passive balun solution will offer some advantages over the other alternatives.

Baluns with the frequency band of $2-13 \mathrm{GHz}$ or similar bandwidth are not commercially available and could not be found in the literature either. Therefore, a new wideband compact balun has been developed and is presented in the paper. This design achieves a bandwidth of $3-13.5 \mathrm{GHz}$ (4.5:1) which is wider than any solution published in literature, for example, the bandwidth of 2:1 in [5]. A whole feeding circuitry based on the new wideband baluns for the dual polarized 2-14 GHz Eleven antenna is also presented in the paper.

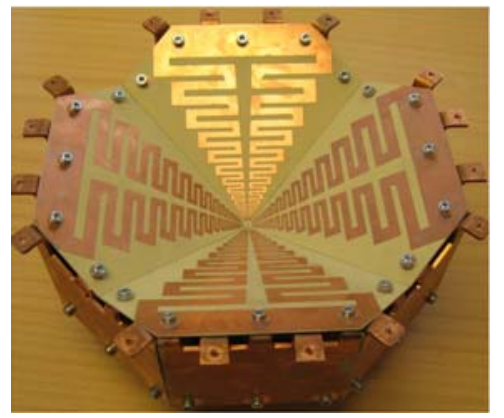

Fig. 1. The 2-14 GHz dual polarized Eleven antenna

\section{New Wideband Balun}

Gibson in 1979 [6] introduced a new frequency independent antenna, the Vivaldi antenna. It is a slot antenna with an exponentially tapered profile. There are several alternatives for designing this type of antenna, but in most cases it consists of one substrate with ground plane on one side and microstrip conductor on the other side. The feeding mechanism for Vivaldi antenna exhibits ultra-wideband characteristics. We have 
in the present paper used this feeding mechanism to develop a new ultra-wideband balun. Fig. 2 shows the geometry for this new wideband balun.

Port 1 of the balun is defined by a $50 \mathrm{ohm}$ microstrip line on the lower side of a dielectric substrate, see Fig. 2. Then, this micro-strip line is coupled to the slot on the upper side of the substrate via a circular slot-line cavity. The latter

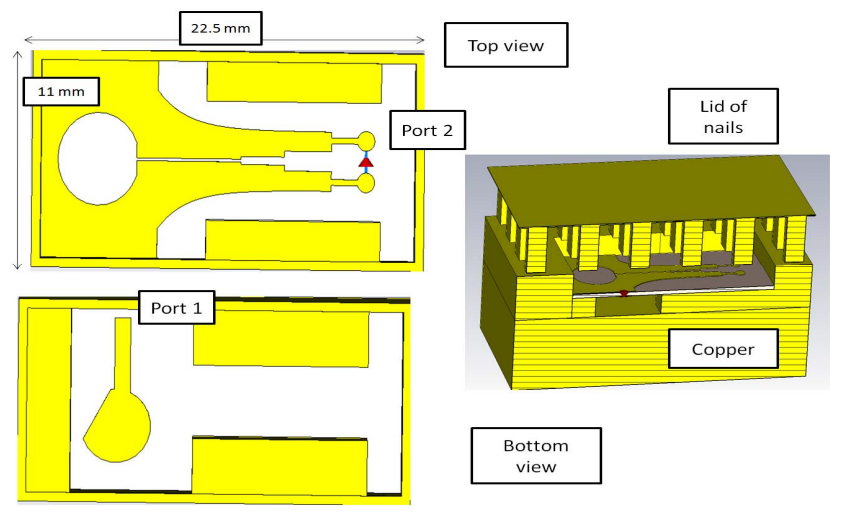

Fig. 2. Top and bottom view of CST model of the new balun, to the right with a lid nails. provides a wideband solution for the coupling. A three-step impedance transformer is used between the slot and the differential coupled microstrip line at port 2 of the balun. The differential impedance of the coupled microstrip line at port 2 is chosen to $200 \mathrm{ohms}$, which is the average input impedance of the Eleven antenna. The substrate for the balun board is Rogers TMM3 with a permittivity of 3.72 .

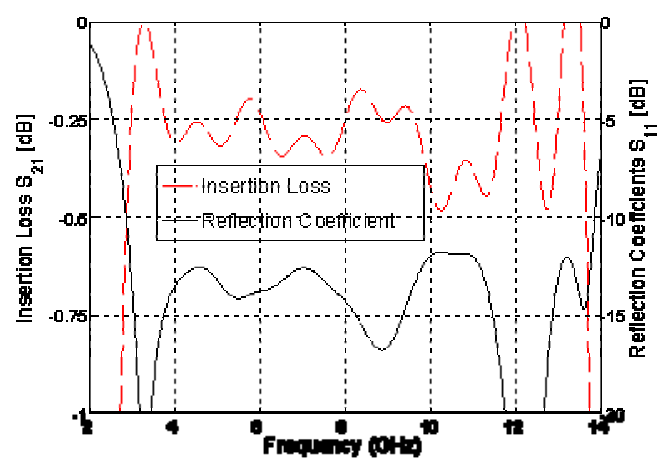

Fig.3. Simulated $S$ parameters of the new balun

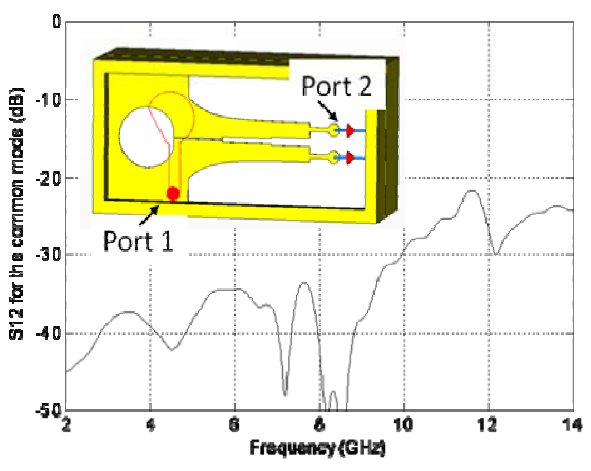

Fig. 4. Common mode suppression of the new balun

The design of the balun was carried out by parameter study and dimension tuning for the following parameters: diameter of the slot-line cavity, size of the microstrip line stub, taper profile at the outer side of the transform, size and number of steps of the slot line transformer. The parameter study and dimension tuning was done by simulations using CST MS [7]. Some design guidelines can be concluded as follows. The diameter of the slot-line cavity and the size of the microstrip line stub affect mainly the performance at the lower end of the frequency band. The taper profile of the slot line affects the performance at the higher end of the band, and the dimensions of the steps of the slot line transformer affect the bandwidth.

The new balun is a multi-layer component; see the side view in Fig.2. In order to avoid creating resonant modes between the ground plane and the bottom metal plate in the operating frequency band, it is required to have a small size of the balun. However, on the other hand, a small size of the balun degrades the performance at low frequencies. An acceptable trade-off has been achieved by the parameter study and dimension tuning in 
this work. The upper plate (lid) is an artificial perfect magnetic conductor (PMC) plate, realized by using the Lid of Nails technology, which suppress all cavity modes between the ground plane and the lid of nails, and between the bottom metal plate and the lid of nails. For the detail of this, please refer to [8][9].

Fig. 3 shows the simulated performance, and the size of the new balun is given in Fig. 2. It can be seen that the reflection coefficient of the new balun is below $-10 \mathrm{~dB}$ over two octaves bandwidth from 3 to $13 \mathrm{GHz}$, the ohmic loss is very low and the size is very compact.

One additional advantage of this balun is that it has a good common mode suppression, which is more than $20 \mathrm{~dB}$, see Fig. 4. Such a high level of suppression can be explained by the geometry of the balun, which rejects common mode due to the direction of the electric field travelling along the slot.

\section{Eleven Feed Descrambling Circuit using New Wideband Baluns}

Four of the new wideband baluns are used in building up a descrambling circuitry for the Eleven feed to reduce from 4 twin line ports to two single-ended ports. The baluns are soldered to four pairs of two-wire lines that pass through the ground plate and connect to the four antenna petals. The overall dimension of the device is $52 \mathrm{~mm} \times 52 \mathrm{~mm}$, which is very compact. Computed S parameters by using CST MS of the complete descrambling circuit connected to $200 \mathrm{ohm}$ loads and connected to the real Eleven antenna petals are shown in Fig. 5. A reflection coefficient of below $-8 \mathrm{~dB}$ over $3-13 \mathrm{GHz}$ for the whole Eleven antenna including the descrambling board has been achieved theoretically.

The descrambler board is in the process of being manufactured, and the measured results including the Eleven feed will be presented at the conference.

\section{Conclusions}

A new two octaves bandwidth balun is presented in the paper, which has a bandwidth of $3-13 \mathrm{GHz}$ with a very compact size, $-10 \mathrm{~dB}$ input reflection coefficient, low ohmic loss and large common mode suppression. A new descrambler circuit for the $2-13 \mathrm{GHz}$ Eleven feed has been designed by using the new baluns, reducing from 4 twin-wire ports to 2 single-ended ports, one per polarization. The reflection coefficient of the whole Eleven antenna with the new descrambling circuit is below $-8 \mathrm{~dB}$ over the frequency range of 3-13 GHz. Further improvement of the bandwidth of the new balun will be emphasized in the future work.

\section{Acknowledgment}

This work has been supported in part by The Swedish Foundation for Strategic Research (SSF) within the Strategic Research Center Charmant.

\section{References}

[1] P.Hall, The SKA: An engineering perspective, Springer, 2005. 
[2] A. Niell, A. Whitney, W. Petrachanko, W. Schluter, N. vandenberg, H. Hase, Y. Koyama, C. Ma, H. Schuh, G. Tuccari, "VLBI 2010 current and future requirments for Geodetic VLBI systems", IVS WG3 report, 2005, http://ivscc.gsfc.nasa.gov/about/wg/wg3.

[3] R. Olsson, P.-S. Kildal, S. Weinreb, "The Eleven antenna: a compact low-profile decade bandwidth dual polarized feed for reflector antennas", IEEE Trans. Antennas Propagat., vol. 54, no. 2, pt. 1, pp. 368-375, Feb. 2006.

[4] J. Yang, M. pantaleev, P.-S. Kildal, Y. Karadikar, L. Helldner, B. Klein, N.Wadefalk, "Cryogenic 2-13 GHz Eleven feed for reflector antennas in future wideband radio telescopes", submitted to IEEE Trans. Antennas Propagat, Dec. 2009.

[5] M. Karlsson and S. Gong, "Circular dipole antenna for mode 1 UWB radio with integrated balun utilizing a flex-rigid structutr," IEEE Trans. Antennas Propagat., vol. 57, no. 10, pp.2967-2971, Oct. 2009.

[6] P. J. Gibson, "The Vivaldi aerial," proc. 9th Europain Microwave Conf., pp.101-105, 1979.

[7] CST Microwave Studio: http://www.cst.com/

[8] E. Rajo-Iglesias, A. U. Zaman, P.-S. Kildal, "Parallel plate cavity mode suppression in microstrip circuit packages using a lid of nails", IEEE Microwave and Wireless Components Letters, vol. 20, no.1, pp.31-33, Jan. 2010.

[9] A. U. Zaman, J. Yang, and P.-S. Kildal, "Using lid of pins for packaging of microstrip board for descrambling the ports of Eleven antenna for radio telescope applications", submitted to IEEE Antennas Propag. Soc. Symp., 2010.

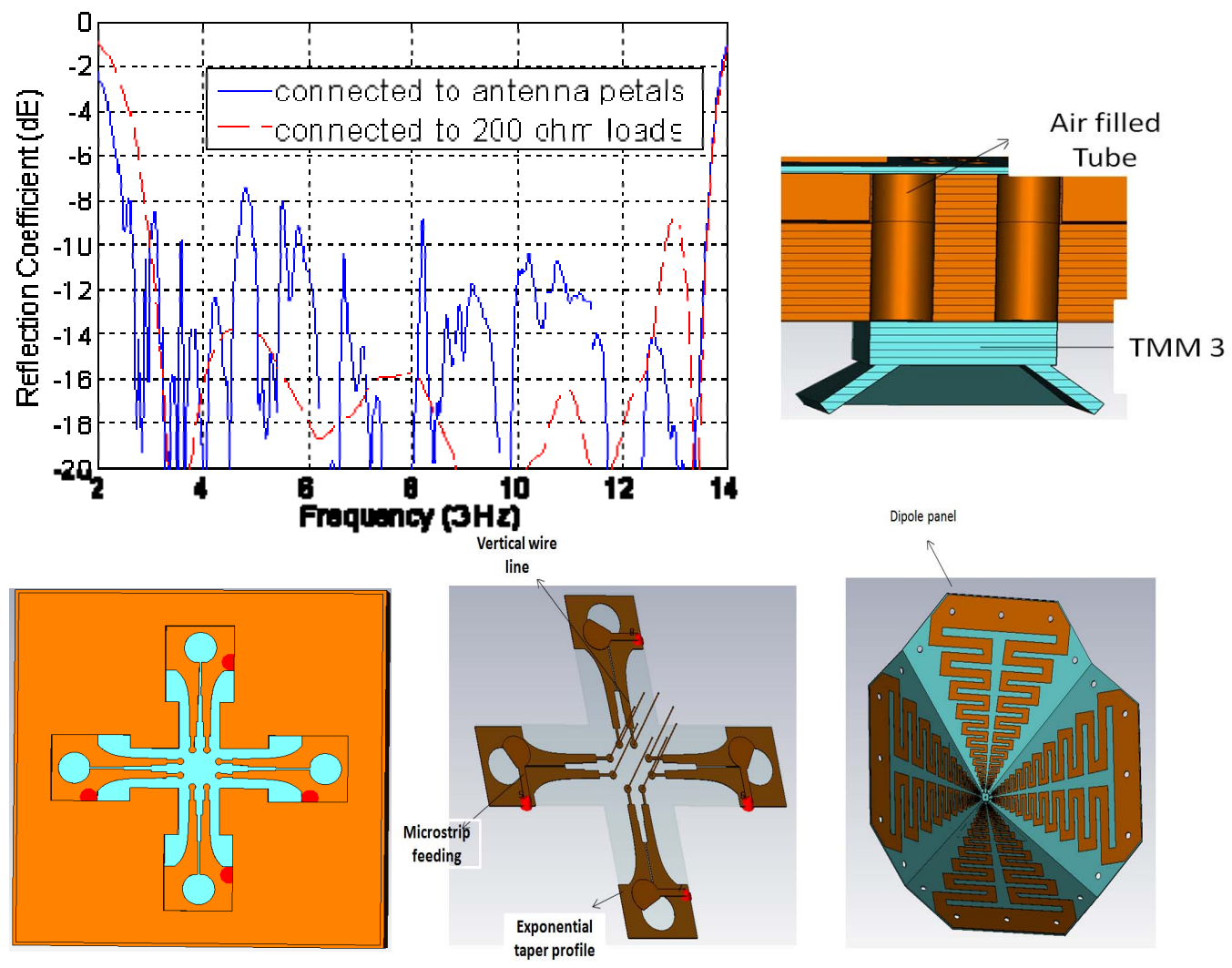

Fig. 5 . Reflection coefficient for Descrambler board including twin lead transition through ground plane and connected to $200 \mathrm{ohm}$ loads (dashed red) or real Eleven antenna petals (solid blue) (upper left), the geometry of twin lead transition through ground plan (upper right), top view of descrambler circuit (lower left), and some views of the descrambling board and the Eleven petals (lower middle and right). 